\title{
Numerical Simulation of High-Speed Impact on Regolith Using Compressible and Non-Expanding Fluid Model
}

\author{
By Kojiro SUZUKI ${ }^{1)}$ \\ ${ }^{1)}$ Department of Advanced Energy, The University of Tokyo, Kashiwa, Japan
}

(Received June 21st, 2017)

\begin{abstract}
The "Compressible and Non-Expanding (CNE)" fluid model was applied for simulation of a high-speed impact on a regolith-like target material, in which irreversible compression occurs. The equation of state for the CNE fluid is composed of the irreversible compression curve and the reversible elastic curves. For numerical simulation with the finite volume method, we developed the Riemann solver, whose fundamental solutions are the combination of the shock waves, the elastic waves, the contact discontinuities and the boundary between the regolith and the vacuum. To track the boundary by the finite volume method, the volumetric fraction function and the cell pattern identification method were introduced. To demonstrate that the present CNE fluid model is promising for the simulation of high-speed impact, the crater formation on the regolith-like target was simulated by the two-dimensional analysis and the result was qualitatively reasonable. The effects of the size (depth) of the sand box and the non-uniformity of the target material were investigated, assuming the one-dimensional approximation on the centerline of the impact.
\end{abstract}

Key Words: Impact, Regolith, Compressible Flow, Numerical Simulation

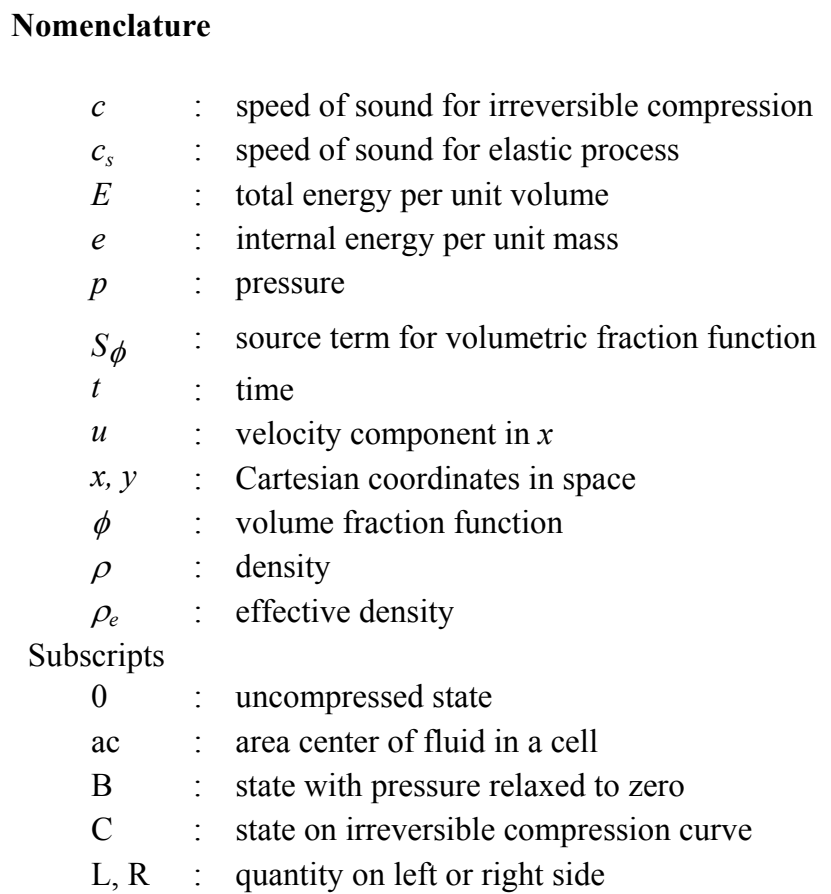

\section{Introduction}

In the solar-system exploration, understanding of high-speed impact phenomena on the regolith-like material is important from a viewpoint of not only planetary science but also spacecraft engineering. For example, modeling of the penetration dynamics was one of the key problems for the development of the penetrator into the lunar regolith in LUNAR-A mission. ${ }^{1)}$ In Hayabusa2 mission, a small kinetic impactor is expected to reveal the properties of the interior of an asteroid. ${ }^{2)}$ Essentially, the impact phenomena of granular materials should be explained as collective phenomena of regolith particles. For example, the Distinct Element Method (DEM) was successfully applied for the numerical simulation of the impact cratering problem. ${ }^{3)}$ However, the phenomena of individual particles are quite complicated and large computational resource is required for the analysis. Such approach is not suitable for solving the engineering problems, most of which are set from a macroscopic viewpoint. In that sense, a macroscopic fluid model to appropriately describe the dynamics of granular materials is expected to become a powerful tool for such problems.

From a macroscopic viewpoint, the behavior of the regolith can be described as a kind of compressible fluid. In fact, the empirical formula to calculate the force acting on the LUNAR-A penetrator body was successfully developed based on the Newtonian theory, ${ }^{4}$ ) which is originally the method to predict the pressure on the surface of the body flying in the atmosphere at a hypersonic speed. ${ }^{5)}$ In the case of the LUNAR-A penetrator, the empirical formula was derived and validated by using a large amount of the experimental data. However, such high-speed impact experiments require a large amount of time and cost to be conducted. If some part of the impact experiments could be replaced by the numerical simulation, the time and cost for developing impact probes would be significantly reduced. Of course, such simulation will be also helpful to understand what happens under the ground from a phenomenological viewpoint.

Considering that the density of the regolith is increased by the compression at the impact, its dynamics is expected to be described as a kind of compressible gas. When the regolith is compressed by the impactor, its density increases and the repulsive force is generated like pressure of the gas. However, unlike usual gas, once the compressive load is removed, its 
density does not decrease back to the initial one, while the pressure vanishes. The regolith exists in vacuum environment without expansion. But such phenomena never happen for usual compressible fluid. Consequently, we need the fluid model, in which the expansion wave does not appear in its flow. To describe such properties, the compressible and non-expanding (CNE) fluid model was proposed. ${ }^{6)}$ In the present study, the improved solver with the finite volume method was used to analyze the one- and two-dimensional impact problems on the regolith. We only considered the situation that the regolith-like material exists in the vacuum. The interaction with the atmospheric gas was not considered.

The present method is categorized into the shock codes, in which the conservation of the mass, momentum and energy is numerically solved. Various shock codes like AUTODYN and iSALE have been developed so far, and are known to provide reasonable results for the impact cratering problems. ${ }^{7)}$ However, the difficulty still remains in describing the feature, which is originated from the behavior of individual particles in the regolith. That is, when contacting particles pushing each other are separated, the repulsive force acting on them instantaneously disappears. The present method is expected to have an advantage, because such asymmetric nature of compression and expansion is clearly considered in the mathematically well-defined framework of the Riemann solver. On the other hand, the material is assumed to be deformed like a fluid and the strength model, which is considered in some shock codes, ${ }^{7)}$ is not included for simplicity of the formulation.

In the present study, the CNE fluid model was applied to the high-speed impact problems on the regolith-like material. The major objectives are:

1) To develop the one- and two- dimensional computational codes to simulate the impact phenomena of the regolith-like material by using the CNE fluid model,

2) To demonstrate that the obtained solutions for the crater formation problem are qualitatively reasonable,

3) To clarify the effect of the sand box size and the uniformity of the regolith on the impact phenomena.

Because the CNE model is still under development for application to the practical problems, we confine ourselves to qualitative discussion on the results.

\section{CNE Fluid Model and Method of Analysis}

\subsection{CNE fluid model}

The essential features required for the fluid model to meet the present objectives are

a) the irreversible compression,

b) the absence of expansion wave,

c) the ability to form the crack without producing any pulling force in it, and

d) the ability to coexist with the vacuum without expansion.

To satisfy all the above requirements, the compressible and non-expanding (CNE) fluid model was proposed. The detail of the model and the method of the analysis for flows of the CNE fluid are explained in Ref. 6). In the present paper, the summary and the improvement after that are described.
The equation of state of the CNE fluid is composed of the irreversible compression curve and the reversible (elastic) process indicated by the line $\mathrm{BC}$ (or $\mathrm{B}^{\prime} \mathrm{C}^{\prime}$ ) as shown in Fig. 1. At any compressed state, the fluid can relax the pressure to zero along the line similar to $\mathrm{BC}$, when the compressive force is removed. In the present study, for simplicity of analysis, the liner models with the constant speed of sound were assumed for both the irreversible compression:

and the elastic process :

$$
p=c^{2}\left(\rho-\rho_{0}\right)
$$

$$
p=c_{s}^{2}\left(\rho-\rho_{B}\right) .
$$

The density at the state $\mathrm{B}$, in which the pressure is relaxed to zero, is given by:

$$
\rho_{B}=\rho-p / c_{s}^{2} .
$$

The relaxation of the pressure occurs at the maximum possible speed to avoid the formation of expansion wave. Then the speed of sound for the elastic process must be much larger than that for the irreversible compression. Because an infinitely large speed of sound cannot be used for the actual computation, the present model allows the fluid to slightly expand at a large but finite speed of sound. The hardening effect, by which the pressure more rapidly increases against the compression for the fluid with higher density, can be described by assuming a variable speed of sound model for the CNE fluid, though the constant speed of sound model was set in the present study for simplicity of analysis.

Though the pressure is given by a function of only the density as shown in Fig. 1, another state quantity is necessary in order to uniquely determine the density and pressure because of the hysteresis behavior of compression described in the CNE fluid model. We introduce the internal energy per unit mass as the work done by the pressure:

$$
e=-\int_{\rho_{0}}^{p} p d\left(\frac{1}{\rho}\right)=\int_{\rho_{0}} \frac{p}{\rho^{2}} d \rho,
$$

which is analytically calculated for the linear model as:

$$
e=c^{2}\left(\ln \frac{\rho_{C}}{\rho_{0}}+\frac{\rho_{0}}{\rho_{C}}-1\right)-c_{s}^{2}\left(\ln \frac{\rho_{C}}{\rho}+\frac{\rho_{B}}{\rho_{C}}-\frac{\rho_{B}}{\rho}\right) .
$$

To consider the presence of the vacuum and the formation of the crack, we introduce the volumetric fraction function $\phi$ after the Volume of Fluid (VOF) method. ${ }^{8)}$ Finally the conservation laws for the $\mathrm{CNE}$ fluid in the one-dimensional problems are given by:

$$
\frac{\partial U}{\partial t}+\frac{\partial F}{\partial x}=\left(\begin{array}{c}
S_{\phi} \\
0 \\
0 \\
0
\end{array}\right), U=\left(\begin{array}{c}
\phi \\
\rho \\
\rho u \\
E
\end{array}\right), F=\left(\begin{array}{c}
\phi u \\
\rho u \\
\rho u^{2}+p \\
(E+p) u
\end{array}\right),
$$

where $E$ is the total energy per unit volume:

$$
E=\rho\left(e+\frac{1}{2} u^{2}\right),
$$

and the viscous friction force is not considered in the present study. The source term for the volumetric fraction function is a cut-off filter, which is necessary to keep the value of $\phi$ in the range of $0 \leq \phi \leq 1$. 


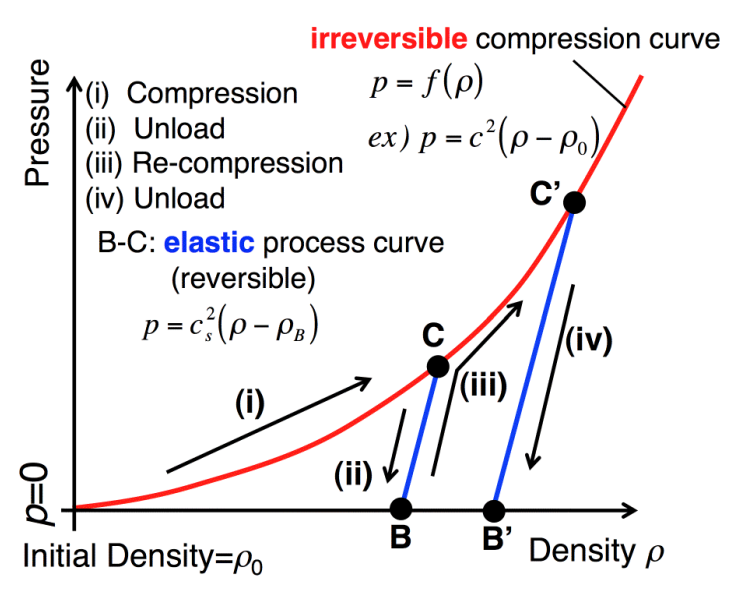

Fig. 1. Equation of state for CNE fluid model.

\subsection{Solution method}

To solve the flow of the CNE fluid, both the fluid surface tracking, in other words, the motion of the boundary between the fluid and the vacuum, and the change in the fluid flow must be simultaneously calculated in the framework of the finite volume method.

In the cell, where the boundary exists, the value of $\phi$ satisfies $0<\phi<1$. In the case of the one-dimensional problem, the ratio of the length of the fluid in a cell to the cell size equals to the volumetric fraction, and four types of the cell pattern are possible as shown in Table 1. For convenience of calculation, we also considered the location of the area center normalized by the cell size in each cell. Figure 2 explains how the formation of the crack is described. When the fluid moves to the right and the speed in the cell i-1 is smaller than that in the cells $i$ and $i+1\left(u_{\mathrm{i}}=u_{\mathrm{i}+1}\right.$ in the figure $)$ at the time step $\mathrm{n}$, the volume flux coming from the cell $\mathrm{i}-1$ to the cell $\mathrm{i}$ between the time steps $n$ and $n+1$ is smaller than that going out of the cell $i$ to the cell $i+1$ as shown in the step 2) in Fig. 2. As a result, the volumetric fraction becomes smaller than 1 . The area center of the cell i moves to the right and $x_{\mathrm{ac}}$ becomes larger than 0.5 . At the time step $n+1$, the pattern of the cell $\mathrm{i}$ is classified into the pattern 3 in Table 1. That is the initiation of the crack formation. As the time step proceeds, $\phi$ and $x_{\mathrm{ac}}$ continue to decrease and increase, respectively (see the step 3 in Fig. 2). That corresponds to the expansion of the crack. Finally the volume flux coming from the cell $i-1$ to the cell $\mathrm{i}$ becomes larger than the volume left in the cell $i$ to go to the cell $i+1$ for the next time step. Then at the next step, the area center jumps to the left of the cell center (the step 4 in Fig. 2), and the volumetric fraction of the cell $\mathrm{i}+1$ becomes smaller than 1 (the step 5). At this time step, the crack spreads in the cells $i$ and $\mathrm{i}+1$. In the above process, the flow quantities in the bar cell of the pattern 2 or 3 must be the same as those in the full $(\phi=1)$ cell that connects the bar cell. In this method, the mass is exactly conserved, while some error appears in the conservation laws for the momentum and the energy. This is because the shape of the boundary between the fluid and the vacuum is described in a simplified way as shown in Table 1.

The Euler equations (6) were numerically solved by the Godunov method ${ }^{9)}$ with the second-order-accuracy MUSCL scheme. ${ }^{6}$ For the Godunov method, the exact Riemann solver is necessary. The fundamental solutions are summarized in terms of the densities on the left and right hand sides of the contact discontinuity as shown in Fig. 3. The fundamental solutions are composed of the shock waves, for which the solution is found on the irreversible compression curve in Fig. 1 , the elastic waves, for which the solution is found on the elastic process curve, the contact discontinuities and the contact surfaces to the vacuum, which allows the CNE fluid to form cracks in it instead of the expansion wave. At the contact discontinuity to the vacuum, the pressure of the fluid must be zero and the density is in the state B in Fig. 1. The quantities on both sides of these waves are calculated by considering the conservation of the mass and the momentum across the wave. The energy is used to determine the pressure on the left and right sides of the Riemann problem. The detailed formulation of the solutions and their classification conditions are given in Ref. 6) except the modification for the case (2) in Fig. 3. In the case (2), we assume the solution, where the elastic wave is formed in front of the shock wave, while the shock wave is assumed to be formed without such precursor wave in Ref. 6), because even a small disturbance causes the precursor wave and the situation assumed in Ref. 6) is hardly realized. In the case (2), the densities on the left side and the right side of the contact discontinuity $\rho_{\mathrm{L}}^{*}$ and $\rho_{\mathrm{R}}^{*}$ are determined by the relation:

$u_{L}-u_{R}=c_{s} \frac{\rho_{C, R}-\rho_{R}}{\sqrt{\rho_{C, R} \rho_{R}}}+c \frac{\rho_{R}^{*}-\rho_{C, R}}{\sqrt{\rho_{C, R} \rho_{R}^{*}}}+c \frac{\rho_{L}^{*}-\rho_{C, L}}{\sqrt{\rho_{C, L} \rho_{L}^{*}}}+c_{s} \frac{\rho_{C, L}-\rho_{L}}{\sqrt{\rho_{C, L} \rho_{L}}}$

and the relation from the pressure balance across the contact discontinuity:

$$
\rho_{L}^{*}=\rho_{R}^{*} \text {. }
$$

The case (2) solution exists when the compression occurs at the cell interface and the velocity difference is high enough to satisfy the relation:

$$
u_{L}-u_{R} \geq c_{s} \frac{\rho_{C, L}-\rho_{L}}{\sqrt{\rho_{C, L} \rho_{L}}}+c \frac{\left|\rho_{C, L}-\rho_{C, R}\right|}{\sqrt{\rho_{C, L} \rho_{C, R}}}+c_{s} \frac{\rho_{C, R}-\rho_{R}}{\sqrt{\rho_{C, R} \rho_{R}}}>0 .
$$

The numerical flux at the cell interface is calculated from the fundamental solution of the Riemann problem.

To describe the hardening effect, the speed of sound must vary with the density and the equation of state becomes non-linear. The solution of the Riemann problem for the CNE fluid with the non-linear equation of state can be obtained in the same way as in Ref. 6), when the pressure monotonically increases with the density on the compression curve. However, the simple analytical formulation as seen in Eqs. (5) and (8) is not available and the numerical procedure is required.

All the cell patterns considered in the two-dimensional problem are summarized in Table 2. The extension of the one-dimensional solver to the two-dimensional problem was made by the fractional time step method ${ }^{9}$ with the second-order accuracy in time. The solution at the $n+1$ time step is obtained by a series of the one-dimensional operations:

$$
U^{n+1}=L_{x}^{1 / 2} \cdot L_{y}^{1} \cdot L_{x}^{1 / 2} \cdot U^{n}
$$

where $L_{x}$ and $L_{y}$ denote the one-dimensional operation in $x$ and $y$, respectively, and the superscript describes the fraction of 
the time step to proceed.

Table 1. Cell patterns for one-dimensional problem.

\begin{tabular}{l|l|l}
\hline Pattern No.: Name & Image & $\begin{array}{l}\text { Normalized } \\
\text { Area Center }\end{array}$ \\
\hline 0: vacant, $\phi=0$ & $\square$ & N/A \\
\hline 1: full, $\phi=1$ & & $x_{\mathrm{ac}}=0.5$ \\
\hline 2: bar (left), $0<\phi<1$ & $\square$ & $x_{\mathrm{ac}}<0.5$ \\
\hline 3: bar (right), $0<\phi<1$ & $\square$ & $x_{\mathrm{ac}}>0.5$ \\
\hline
\end{tabular}

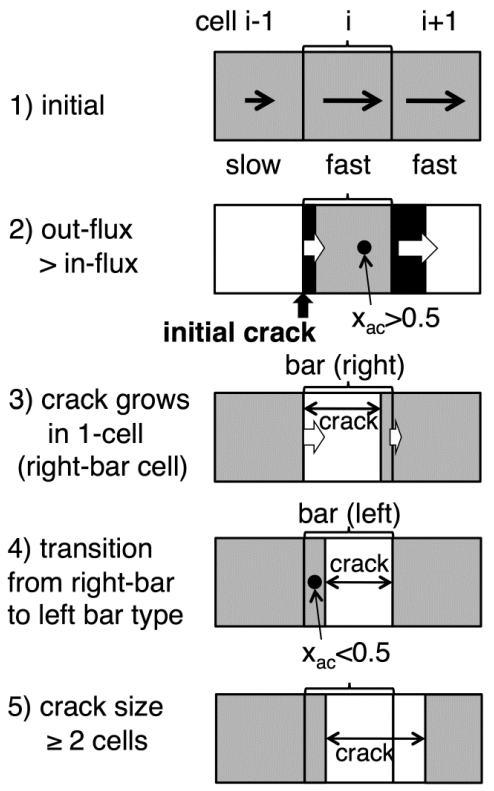

Fig. 2. Formation of crack in one-dimensional problem with finite volume method and volumetric fraction function.

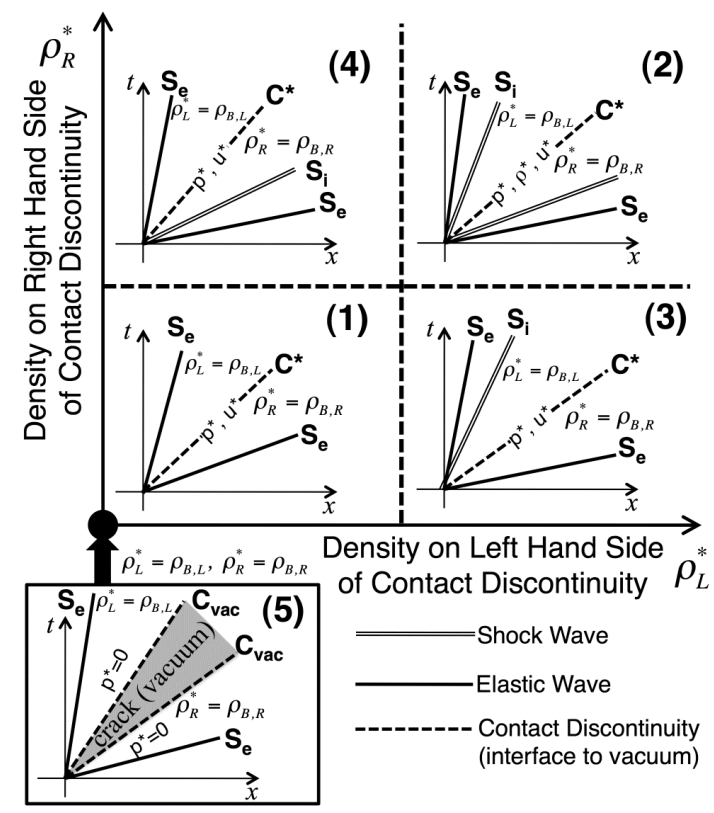

Fig. 3. Fundamental solutions of one-dimensional Riemann problem for CNE fluid model.

\begin{tabular}{l|c|l}
\multicolumn{3}{c|}{ Table 2. Cell patterns for two-dimensional problem. } \\
\hline Pattern No.: Name & Image & $\begin{array}{l}\text { Normalized Area } \\
\text { Center }\end{array}$ \\
\hline 0: vacant $(\phi=0)$ & $\square$ & N/A \\
\hline 1: full $(\phi=1)$ & & $x_{\mathrm{ac}}=0.5, y_{\mathrm{ac}}=0.5$ \\
\hline 2: bar (left) & $\square$ & $x_{\mathrm{a}}<0.5, y_{\mathrm{ac}}=0.5$ \\
\hline 3: bar (bottom) & $\square$ & $x_{\mathrm{ac}}=0.5, y_{\mathrm{ac}}<0.5$ \\
\hline 4: bar (right) & $\square$ & $x_{\mathrm{ac}}>0.5, y_{\mathrm{ac}}=0.5$ \\
\hline 5: bar (top) & $\square$ & $x_{\mathrm{ac}}=0.5, y_{\mathrm{ac}}>0.5$ \\
\hline 6: corner (left-bottom) & $\square$ & $x_{\mathrm{ac}}<0.5, y_{\mathrm{ac}}<0.5$ \\
\hline 7: corner (right-bottom) & $\square$ & $x_{\mathrm{ac}}>0.5, y_{\mathrm{ac}}<0.5$ \\
\hline 8: corner (right-top) & $\square$ & $x_{\mathrm{ac}}>0.5, y_{\mathrm{ac}}>0.5$ \\
\hline 9: corner (left-top) & $\mathbf{}$ & $x_{\mathrm{ac}}<0.5, y_{\mathrm{ac}}>0.5$ \\
\hline
\end{tabular}

\section{Results and Discussion}

\subsection{Two-dimensional impact problem}

As a typical problem about the high-speed impact on the regolith, we solved the two-dimensional crater formation problem as defined in Fig. 4. The hypothetical regolith model with the density in the uncompressed condition, the speed of sound for irreversible compression and that for the elastic process $1800 \mathrm{~kg} / \mathrm{m}^{3}, 150 \mathrm{~m} / \mathrm{s}$ and $3000 \mathrm{~m} / \mathrm{s}$, respectively, was assumed. The two-dimensional rectangular target was set with the initial density $1.2 \rho_{0}$, which means the regolith was pre-compressed and then the pressure was relaxed to the state $\mathrm{B}$ in Fig. 1. The impactor was a two-dimensional circular cylinder made from the pre-compressed fluid with the density $1.2 \rho_{0}$. The impact velocity was $400 \mathrm{~m} / \mathrm{s}$ normal to the target surface. The uniform mesh with the size $0.01 \mathrm{~m}$ was used. The time step size was determined from the CFL number 0.02 based on $c_{\mathrm{s}}$ and it was $6.66 \times 10^{-5} \mathrm{~ms}$. Such small time step was necessary to keep the numerical stability at the calculation. The presence of the elastic process with an extremely high rate of change in pressure with respect to the density change makes the calculation of the CNE fluid quite sensitive to the numerical error. On the wall of the sand box in the region of $y<0$, the rigid boundary condition, in which the normal velocity is forced to become zero and the fluid is allowed to move in the tangential direction, was applied. The flow quantities on the rigid boundary were calculated by considering the mirror image beyond the boundary. Above the sand box $(y>0)$, the no-reflection and free-outgoing boundary condition was considered by assuming the presence of the vacuum beyond it.

The distributions of the pressure and the effective density just after the impact $(t=0.5 \mathrm{~ms})$ are shown in Figs. 5 and 6 , respectively. The density was evaluated by the effective density defined by:

$$
\rho_{e}=\rho / \phi,
$$

where the volume occupied by the fluid in a cell is considered. The pressure rise from the state $\mathrm{B}$ to $\mathrm{C}$ (see Fig. 1) propagated at the speed of $c_{s}$, and almost all the target domain was pressurized at $t=0.5 \mathrm{~ms}$. Though the pressure rise quickly 
propagated, the high-density zone was formed only in front of the impactor. The coalescence of the impactor to the target was seen in the density plot.

From the distribution of the effective density at $t=2.5 \mathrm{~ms}$ (Fig. 7) and $5 \mathrm{~ms}$ (Fig. 8), we found how the collapse hole and the zone with high density were formed. A local spike-like pattern on the centerline was caused by numerical instability. At the time $5 \mathrm{~ms}$, it is observed that the rim was formed around the collapse hole due to the presence of the small upward velocity there. In the present analysis, the impactor penetrated deeply under the surface of the target, because the speed of sound was assumed to be constant and the hardening effect was not included. These results imply that the impact phenomena are roughly divided into two stages: 1) rapid deceleration of the impactor and compression of the target, which are accompanied by the shock waves, and 2) slow deformation of the surface caused by induced motion of the regolith. The stage 2) is expected to continue for a long time beyond the present computation time because of the lack of damping force.

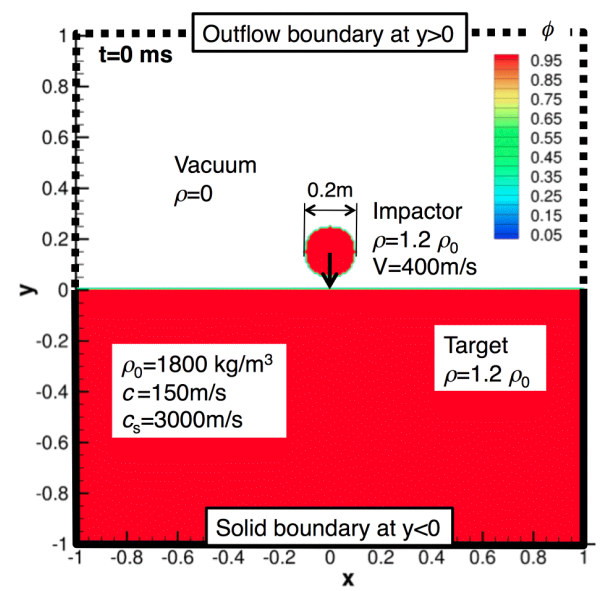

Fig. 4. Problem setting of two-dimensional impact problem.

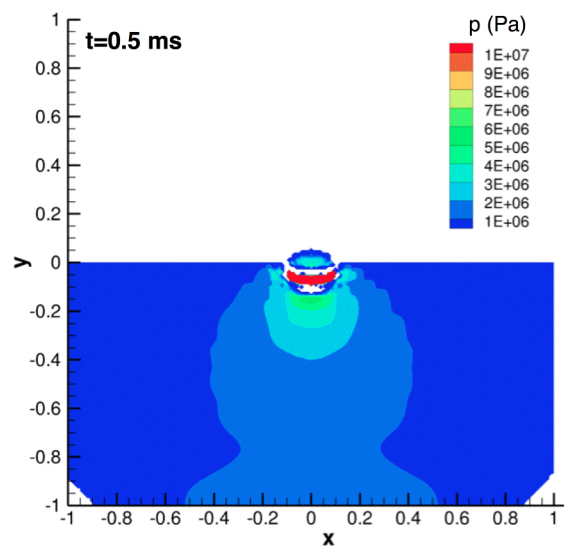

Fig. 5. Distribution of pressure at $t=0.5 \mathrm{~ms}$.

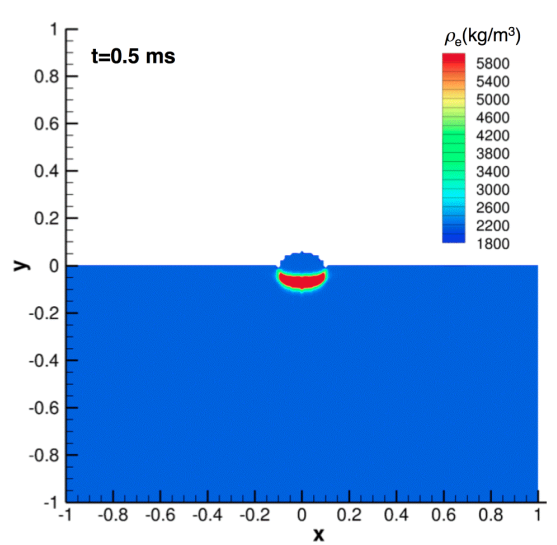

Fig. 6. Distribution of effective density at $t=0.5 \mathrm{~ms}$.

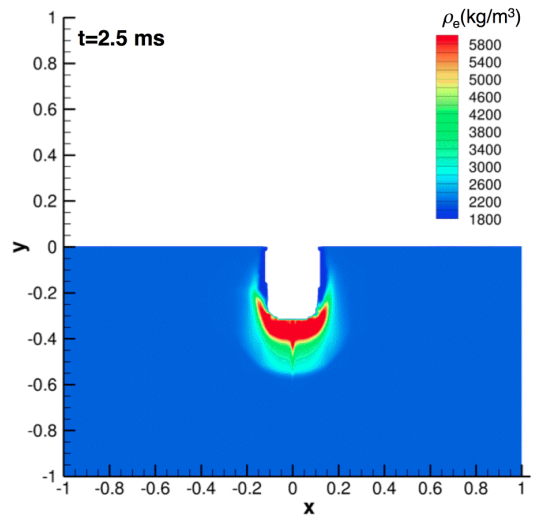

Fig. 7. Distribution of effective density at $t=2.5 \mathrm{~ms}$.

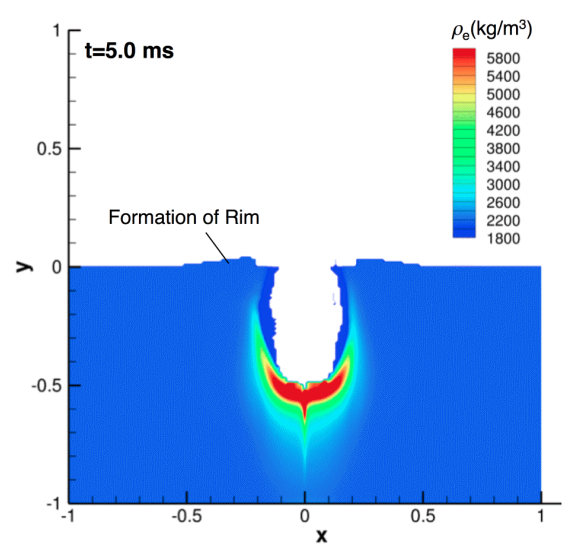

Fig. 8. Distribution of effective density at $t=5 \mathrm{~ms}$.

Considering the symmetry with respect to the centerline $(x=0)$, the one-dimensional impact problem is expected to approximate the phenomena on the centerline in the tow-dimensional crater formation problem. The time-depth map for the effective density by the one-dimensional analysis with the same computational parameters is shown in Fig. 9. The qualitatively similar trend is obtained to the two-dimensional analysis with respect to the crater depth and the density of the compressed regolith near the surface especially at the early stage of the impact. After the time 2.5 $\mathrm{ms}$, the effects of multi-dimensionality and waves reflected at the bottom degrade the quality of the approximation.

At present, the calculation was conducted in the hypothetical two-dimensional condition. After the 
development of the three-dimensional code, the validation will be made by comparison with the experimental data. For example, the results of the impact experiments are available as well as the numerical simulation model for DEM in Ref. 10). For the computational condition to be consistent with the experimental one, the parameters $c$ and $c_{s}$ in the equation of state should be determined beforehand based on the simulation of the compression and unload processes by the DEM analysis with the same model parameters.

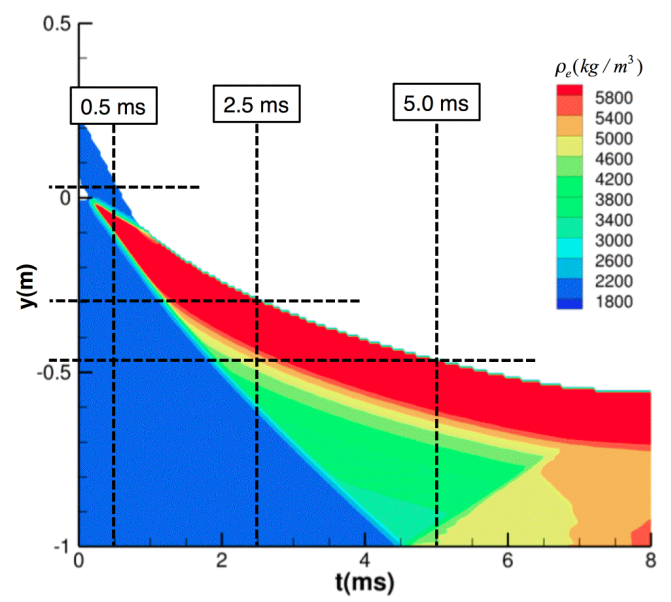

Fig. 9. Time(t)-depth(y) map of effective density for one-dimensional impact problem with the same parameters as two-dimensional crater formation problem.

\subsection{One-dimensional impact problem}

The one-dimensional impact problem is suitable for the parametric study to understand the characteristic features of the CNE fluid model because of its small computation time. As a hypothetical regolith model, the density in the uncompressed condition, the speed of sound for irreversible compression, that for the elastic process were assumed to be $1800 \mathrm{~kg} / \mathrm{m}^{3}, 300 \mathrm{~m} / \mathrm{s}$ and $3000 \mathrm{~m} / \mathrm{s}$, respectively. The computation domain extended from $x=-5.0 \mathrm{~m}$ to $x=1.5 \mathrm{~m}$, where $x$ is the in-depth coordinate. The uniform mesh with the size $0.01 \mathrm{~m}$ was used. The target was set in the region $x \leq 0$. The initial length of the impactor was $0.4 \mathrm{~m}$ and was located from $x=0.8 \mathrm{~m}$ to $x=1.2 \mathrm{~m}$. At the bottom $(x=-5 \mathrm{~m})$, we assumed the rigid wall condition, where the velocity is zero and the other quantities are calculated by assuming their mirror images with respect to the wall. The initial density was $\rho_{0}$ for both the target and the impactor. The initial pressure was zero all over the computational domain. The target was initially at rest and the impact velocity was the computational parameter.

The distribution of the volumetric fraction function on the time-depth map is shown in Fig. 10. The impact velocity was $1000 \mathrm{~m} / \mathrm{s}$. The time integration was made by the Euler explicit scheme with the first-order accuracy in time. The time step size was determined from the CFL number 0.02 based on $c_{\mathrm{s}}$ and it was $6.66 \times 10^{-5} \mathrm{~ms}$. A very small time step was necessary to keep the numerical stability at the calculation as required in the two-dimensional analysis. The coalescence of the impactor with the target after the impact, the collapse of the surface and the deceleration of the collapse speed were reasonably simulated by the present method. Thanks to the step-like distribution pattern assumed in a cell as shown in Table 1, the boundary between the fluid and the vacuum was sharply captured without numerical dissipation. The collapse of the surface stopped at about $t=20 \mathrm{~ms}$, and the fluid motion almost disappears after that time. It should be noted that a small piece of ejecta seems to be injected from the surface at $t=20 \mathrm{~ms}$. This is thought to be initiated by some numerical oscillation. However, such solution pattern shows that the crack formation mechanism in the present method works well as explained in Fig. 2. Some amount of the mass at the surface was lost by the injection of the ejecta and the surface density decreased after that.

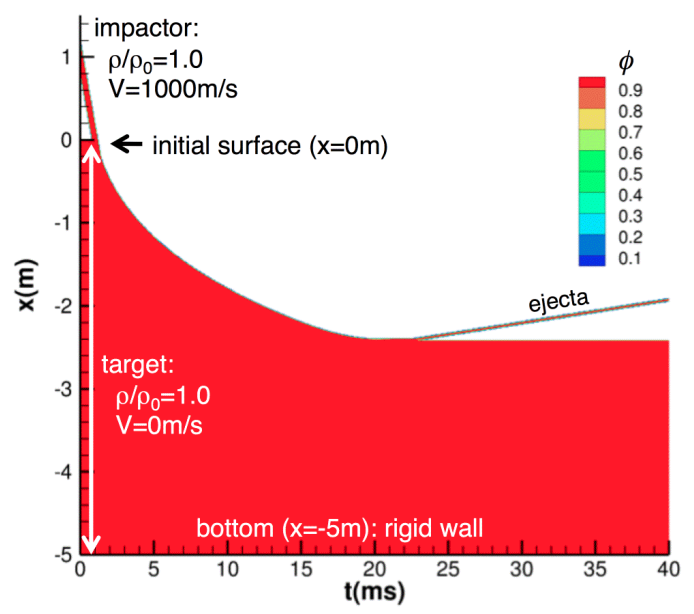

Fig. 10. Time-depth map of volumetric fraction function obtained by one-dimensional impact problem with impact velocity $1000 \mathrm{~m} / \mathrm{s}$, sand box depth $5 \mathrm{~m}$ and uniform target.

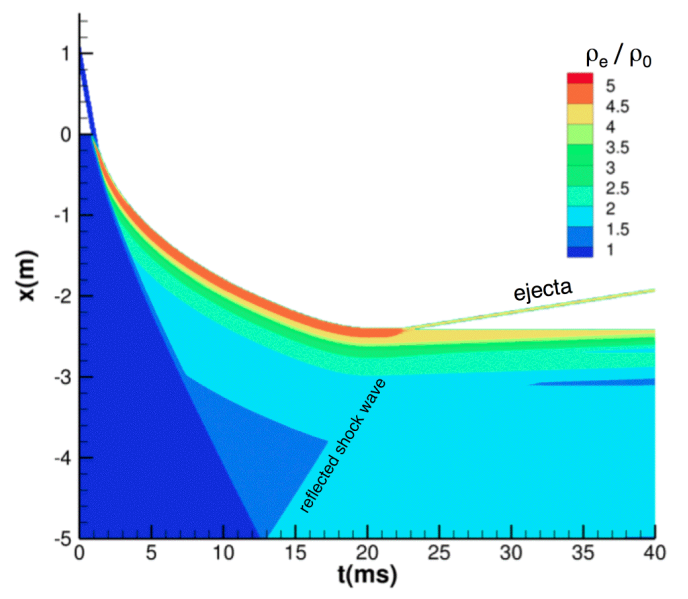

Fig. 11. Time-depth map of effective density obtained by onedimensional impact problem with impact velocity $1000 \mathrm{~m} / \mathrm{s}$, sand box depth $5 \mathrm{~m}$ and uniform target.

The wave diagrams for the effective density and pressure distributions are shown in Figs. 11 and 12, respectively. In the present CNE fluid model, the zone of high density remains on the surface without unphysical expansion to the vacuum. It is clearly seen in Fig. 12 that the shock wave propagated in the target interior and the region with high pressure appeared behind the shock wave. On the other hand, the pressure decreased to the contact surface to the vacuum, where the 
pressure must be zero. The shock wave was reflected at the rigid wall of the sand box bottom. When the reflected shock wave reached the surface, the release of the pressure to zero quickly propagated from the surface to the bottom at the speed of sound for the elastic process $(3000 \mathrm{~m} / \mathrm{s})$. After that, the pressure became zero all over the fluid. The presence of the zone of high pressure behind the reflected shock wave generated the upward velocity for the fluid. After the pressure release, there is no force to accelerate or decelerate the fluid. The fluid, which obtained the velocity before the pressure release, continued to move.

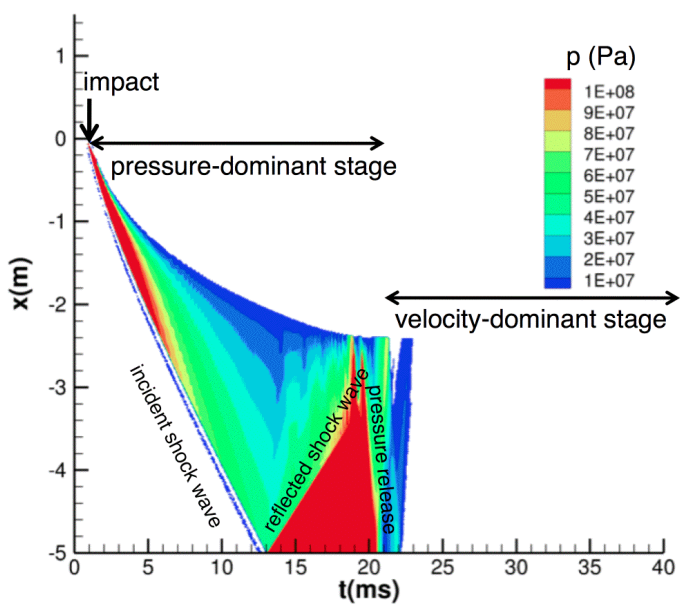

Fig. 12. Time-depth map of pressure obtained by one-dimensional impact problem with impact velocity $1000 \mathrm{~m} / \mathrm{s}$, sand box depth $5 \mathrm{~m}$ and uniform target.

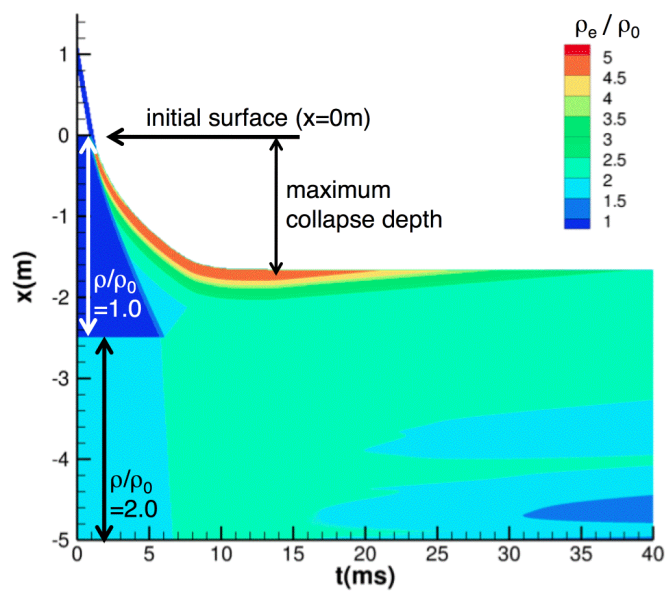

Fig. 13. Time-depth map of effective density obtained by onedimensional impact problem with impact velocity $1000 \mathrm{~m} / \mathrm{s}$, sand box depth $5 \mathrm{~m}$ and two-layer target.

In addition to the case with the uniform target, the target composed of the upper layer at low density $\rho_{0}$ and the lower layer at high density $2 \rho_{0}$ was also analyzed. The distribution of the effective density on the time-depth map for the two-layer target is shown in Fig. 13. The impact velocity was $1000 \mathrm{~m} / \mathrm{s}$. When the incident shock wave reached the boundary of these layers, the reflected shock wave was formed in the upper layer and the elastic wave, which raised the pressure from the state $\mathrm{B}$ to the state $\mathrm{C}$ or some state between $\mathrm{B}$ and $\mathrm{C}$ (see Fig. 1), propagated in the lower layer. The collapse depth for the two-layer target was apparently smaller than that for the uniform target due to the presence of the hard layer in the target. In this case, the fluid continued to slowly move upward because of the presence of high pressure behind the refracted shock wave. The surface slowly moved upward after the maximum collapse depth. The effective density near the surface was decreased by the upward motion of the fluid there.

The variation of the maximum collapse depth with the impact velocity is compared among the cases of the $10 \mathrm{~m}$ bottom, $5 \mathrm{~m}$ bottom, $2.5 \mathrm{~m}$ bottom and the $5 \mathrm{~m}$ bottom with the two-layer target in Fig. 14. To check the grid convergence, the computation using the fine mesh with the mesh size 0.005 $\mathrm{m}$ was made for the case of the sand box depth $5 \mathrm{~m}$. As shown in Fig. 14, the difference between the results by the normal mesh and the fine mesh was smaller than $3 \%$. The collapse depth nonlinearly increases with the impact velocity. The depth for the two-layer target is much smaller than that for the uniform target. The presence of the bottom is necessary to stop the collapse, and the maximum collapse depth increases with the increase in the sand box depth. Those results tell us that we have to be careful to appropriately evaluate the effect of the sand box size and the uniformity of the target material, when we make experimental simulation of the impactor using a sand box with a finite-size.

In the present model, the work done by the pressure is stored in the regolith as the internal energy. Though the rise in the internal energy will cause the rise in the temperature, the physical properties of the CNE fluid are assumed to be independent of the temperature. In the case of a high impact velocity, however, the temperature rise may become unphysical beyond the melting point. In the case of the sand box depth $5 \mathrm{~m}$, the maximum internal energy is about 166000 $\mathrm{J} / \mathrm{kg}$ for the impact velocity $2000 \mathrm{~m} / \mathrm{s}$. Considering that the specific heat of the lunar regolith is $500-800 \mathrm{~J} /(\mathrm{kg} \cdot \mathrm{K}),{ }^{11)}$ the maximum temperature rise is roughly estimated as $200-300 \mathrm{~K}$ and the temperature effect is expected not to be significant. However, it strongly depends on the problem setting. For the present CNE fluid model to be physically valid, the limitation on the impact velocity must be carefully determined depending on the problem setting.

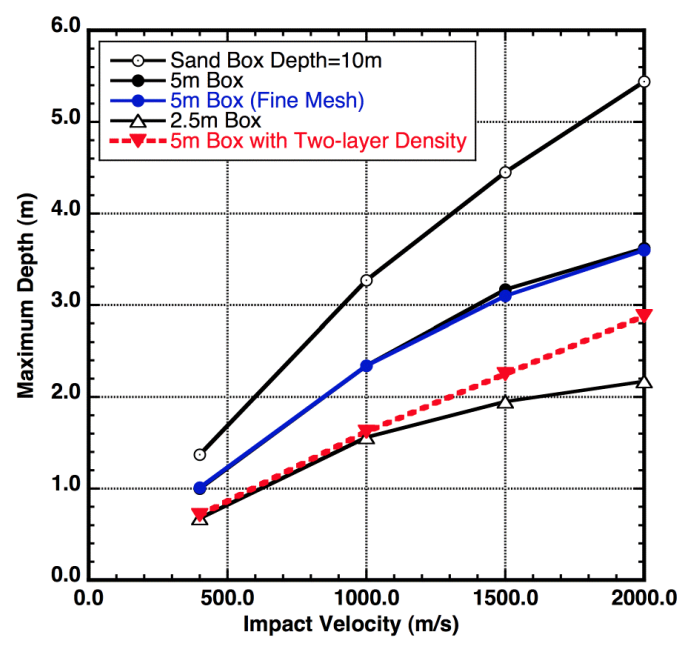

Fig. 14. Effect of sand box depth and uniformity of target on variation of maximum collapse depth with impact velocity. 


\section{Conclusion}

In the present study, the compressible and non-expanding (CNE) fluid model was proposed to use the analysis of high-speed impact problems on the regolith-like material. The major conclusions are as follows:

1) The computational codes to solve the one- and the two-dimensional impact problems were developed by using the CNE fluid model and the Riemann solver for it. In order to accurately capture the deformation of the surface of the material within a framework of the finite volume method, the step-function type distribution of the material in a cell and the cell pattern identification based on the area center of the material in a cell were introduced. The combination of the Riemann solver for the CNE fluid model and the present surface tracking method works well for the impact problems.

2) The present CNE fluid model successfully simulated the irreversible compression. The compressed material with high density remains near the surface of the target after all the events of the impact end and the motion of the fluid stops.

3) The effect of the sand box size and the uniformity of the target material were numerically investigated by solving the one-dimensional impact problems. The results show that the collapse depth strongly depends on them. When we use a small sand box for impact experiments, we may significantly underestimate the penetration depth of the impactor.

\section{Acknowledgments}

This work is supported by Grant-in-Aid for Scientific Research (B) No. 16H04585 of Japan Society for the Promotion of Science.

\section{References}

1) Shiraishi, H., Tanaka, S., Hayakawa, M., Fujimura, A., Mizutani, H., and Suzuki, K.: Dynamical Characteristics of a Planetary Penetrator, Penetrometry in the Solar System II, Graz, pp. 217-232, 2006.

2) Saiki, T., Imamura, H., Sawada, H., Arakawa, M., Kadono, T., Takagi, Y., and Wada, K.: Small Carry-on Impactor of Hayabusa2 and Its Impact Operation, 29th International Symposium on Space Technology and Science, Nagoya, ISTS 2013-d-18, 2013.

3) Wada, K., Senshu, H., and Matsui, T.: Numerical Simulation of Impact Cratering on Granular Material, Icarus, 180(2006), pp. 528-545.

4) Suzuki, K., Abe, T., Higuchi, K., Fujimura, A., and Mizutani, H.: Development of Analysis Model of Penetration Dynamics for LUNAR-A Mission, 20th International Symposium on Space Technology and Science, Gifu, 96-i-02V, 1996.

5) Anderson, Jr., J. D.: Hypersonic and High Temperature Gas Dynamics, McGraw-Hill, New York, 1989, pp. 45-66.

6) Suzuki, K.: Compressible and Non-Expanding Fluid Model for Simulation of Impact Phenomena, AIAA Paper 2016-4107, 2016.

7) Pierazzo, E., Artemieva, N., Asphaug, E., Baldwin, C., Cazamias, J., Coker R., Collins, G. S., Crawford, D. A., Davison, T., Elbeshausen, D., Holsapple, K. A., Housen, R. A., Korycansky, D. G., and Wünnemann, K.: Validation of Numerical Codes for Impact and Explosion Cratering: Impacts on Strengthless and Metal Targets, Meteoritics \& Planetary Science, 43(2008), pp. 1917-1938.

8) Hirt, C. W. and Nichols, B. D.: Volume of Fluid (VOF) Method for the Dynamics of Free Boundaries, J. Comp. Phys., 39(1981), pp. 201-225.

9) Toro, E. F.: Riemann Solvers and Numerical Methods for Fluid Dynamics, Springer-Verlag, Berlin, 1997, pp. 201-220 and pp. 509-549.

10) Nakamura, A. M., Setoh, M., Wada, K., Yamashita, Y., and Sangen, K.: Impact and Intrusion Experiments on the Deceleration of Low-gravity Impactors by Small-Body Regolith, Icarus, 223(2013), pp. 222-233.

11) Hemingway, B. S., Robie, R. A., and Wilson, W. H.: Specific Heats of Lunar Soils, Basalt, and Breccias from the Apollo 14, 15, and 16 Landing Sites between 90 and $350{ }^{\circ} \mathrm{K}$, Proceedings of the Fourth Lunar Science Conference, Vol. 3, pp. 2481-2487, 1973. 